\title{
Research on Event-based Geospatial Data Updating
}

\author{
ZHOU Zhao $^{\text {a, } *, \text { SUN Qun }}{ }^{\text {a }}$, LYU Xiaohua ${ }^{\text {a }}$ \\ a Information Engineering University, zhouzhaochxy@126.com,SUN Qun,yinghong.jia@163.com, LYU Xiaohua, \\ cylvxh_vip@sina.com
}

Keywords: Geospatial data updating, Geographical features spatio-temporal change, Geographical event, Homologous geographical feature matching

\begin{abstract}
:
How to update the geospatial data timely and accurately has become the focus of surveying and mapping. However, an efficient updating system has not been set up so far. Still the updating operations depend on human-computer interaction, which is less efficient, labour-consuming and prone to error. Accordingly, this paper proposes a new eventbased updating method of geospatial data in order to improve the automation and intelligence of data updating. The main contents of the paper are as follows:

On the basis of the five classical categories of spatio-temporal change typology, the paper proposes a new classification which includes create, transform, death, disappear, reappear, split, divide, combine, merge and so on. The above new classification of spatio-temporal change type is more compatible with geospatial data updating.

The paper proposes a new conception about the life cycle of geospatial features which simulates spatio-temporal changing process of the geospatial entity on the world. The life cycle of geospatial features is composed of three stages: emergence, existence and death. The rules of the above life cycle of geospatial features are also set up.

3.The paper gives a definition of geographical events and also sets up the conceptual model of geographical events which is composed of time, location, geospatial feature, geographical event type and procedure .To better meet the demand of geospatial data updating, the geographical events are also reclassified into create event, transform event, death event, disappear event, reappear event, evolution event, split event, divide event, combine event and merge event.

The paper suggests that homologous geographical feature matching and change detecting should be used to deduce spatio-temporal change type and extract geographical events. The thesis intends to set up an up-to-down matching pattern with four layers so as to improve the efficiency and accuracy of matching. Buffer area matching and attribute matching are used to match the point features of the same names. Both the minimum bounding rectangle overlap area and discrete Fréchet distance are used to match the line features of the same names. As for area features, feature overlapping area and Hausdorff distance are employed in matching. In addition, the thesis proposes to use XML to organize and store dynamic updating operation.
\end{abstract}

Under the guidance of event-based geospatial data updating method, the related prototype system has been established and tested in the updating experiments of residence community and roads. 